\title{
Evaluación y mejora de la calidad de los recursos educativos electrónicos en el ámbito universitario español desde un enfoque documental
}

Information-driven quality assessment and improvement of electronic educative resources in the Spanish university environment

\author{
María PINTo MolinA \\ Universidad de Granada, mpinto@ugr.es
}

\begin{abstract}
Resumen
Se aborda la necesidad de evaluación de la calidad de los recursos educativos electrónicos en contextos virtuales como apuesta estratégica para el aprendizaje significativo en un momento de cambio hacia la plena convergencia europea. Se insiste en la necesidad de fomentar el entrenamiento en la competencia evaluadora de profesores y estudiantes y se menciona algunos instrumentos de ayuda para el diagnóstico objetivo de la calidad de los recursos educativos electrónicos para determinar aquellos que reúnan un umbral de excelencia adecuado. Se exponen las líneas maestras del proyecto de investigación y desarrollo EVALUAREED en el marco de la enseñanza superior.
\end{abstract}

Palabras clave: Evaluación de la calidad. Recursos educativos electrónicos. Aprendizaje electrónico. Enseñanza superior. Proyecto EVALUAREED.

\section{Introducción (1)}

Cada vez más cercano el compromiso de construcción del Espacio Europeo de Educación Superior, todos los sistemas universitarios europeos se esfuerzan por alcanzar los objetivos de integración propuestos para el año 2010. Entre las premisas básicas de las directrices de la Declaración de Bolonia, la individualización del aprendizaje y la responsabilidad del estudiante ante su propio aprendizaje, constituyen cuestiones que deben ser asumidas en la renovación de los métodos pedagógicos, en la línea de las teorías constructivistas según las cuales el aprendizaje es visto como un proceso de construcción individual interna de dicho conocimiento (Jonassen, 1991) y, por tanto, los nuevos modelos educativos basados en competencias y habilidades deben reemplazar a los antiguos modelos basados en la enseñanza del profesorado y el estudio pasivo por parte del alumnado.

\begin{abstract}
The need to evaluate the quality of the electronic learning resources in virtual context is analyzed as a strategic bet in pursue of a more significative learning in the current Spanish educational environment, characterized by the change toward the European educational convergence. The need to promote the training in the assessment competence of both students and teachers is stressed. Some tools for the objective quality assessment of the educational electronic resources are presented that can ensure that they reach an adequate level of excelence. The master guidelines of the R\&D project EVALUAREED is presented, specifically oriented to the higher education environment.
\end{abstract}

Keywords: Quality assessment. Electronic resources. Educational resources. E-Learning. Higher education. EVALUAREED project.

La sociedad en la que vivimos, caracterizada por un uso masivo de información y de tecnologías, y que ha sido denominada Sociedad del Conocimiento, precisa de unas estructuras organizativas flexibles en la educación superior, que posibiliten tanto un amplio acceso social al conocimiento como una capacitación personal crítica que favorezca la evaluación e interpretación de la información y la generación del propio conocimiento.

La aplicación cada vez más generalizada de las Tecnologías de la Información y la Comunicación (TIC) al ámbito educativo (TICE) está facilitando la renovación de los métodos pedagógicos y educativos, y la potenciación de nuevos entornos de enseñanza-aprendizaje concebidos como espacios abiertos, flexibles, interactivos y dinámicos, donde se integran de manera coherente todos los elementos que componen estas comunidades virtuales de aprendizaje. Es el llamado aprendizaje en contextos virtuales, que hacen uso de plataformas virtuales de 
aprendizaje cuyo funcionamiento gira en torno a las herramientas tecnológicas. Las TICE proporcionan las herramientas necesarias para llevar a cabo estas nuevas formas innovadoras de aprendizaje y son utilizadas como instrumentos pedagógicos en los nuevos entornos de aprendizaje virtual.

Un entorno de aprendizaje se define como un lugar o una comunidad donde se llevan a cabo una serie de actividades con la finalidad de apoyar el aprendizaje y donde los actores tienen acceso a numerosos recursos electrónicos (Unión Europea, 2004). En el sistema universitario español cada vez más son los centros que incorporan formación a distancia y plataformas virtuales como recursos tecnológicos de enseñanza-aprendizaje abiertos, dinámicos y flexibles. Dentro de la misma Universidad, la enseñanza a distancia apoyada en Internet supone además un complemento muy interesante y útil a las clases presenciales, al utilizar recursos tecnológicos generalmente conocidos por los estudiantes - como las herramientas de comunicación y de participación: foros, chats, correo electrónico -, utilizados ampliamente en su vida cotidiana. El manejar dichos recursos para sus clases los motiva en su aprendizaje. Por otra parte, la implantación del crédito europeo, que computa el trabajo presencial y no presencial del estudiante, abre todo un campo de posibilidades a la enseñanza virtual o semipresencial, con lo cual, el futuro de la enseñanza y la formación a través de Internet es imparable. Efectivamente, un $52 \%$ de las universidades españolas tienen algún tipo de oferta docente a través de Internet, ya sea propia o compartida con otros centros. En España, esta oferta se resume en dos modelos de oferta docente universitaria a través de Internet: campus virtuales - paralelos a la red física de centros presenciales- y la constitución de universidades completamente virtuales, como la Universitat Oberta de Catalunya. Por otro lado, es interesante señalar que las universidades públicas $(61 \%)$ casi doblan el porcentaje de centros privados $(31,5 \%)$ que disponen de alguna oferta formativa virtual.

\section{El aprendizaje autónomo y el protagonismo de los recursos electrónicos educativos}

El desarrollo de Internet y de las tecnologías de la información han alterado el panorama de las fuentes de información y de los sistemas de recuperación inherentes a ellas, debido a la aparición de fuentes documentales completamente nuevas y de aplicaciones de navegabilidad e intercambio de información que han potenciado la interconexión entre usuarios así como el intercambio de información entre ellos. Si la información es una necesidad consustancial al desarrollo cognitivo y social y las competencias para su control y gestión son inherentes a todo proceso formativo, el enriquecimiento y la mejora en las aplicaciones disponibles para ello representa un avance que toda formación ha de tomar en consideración. Rumsey (2008) subraya la importancia fundamental que el aprendizaje de las habilidades informacionales representa para todo profesor y estudiante, destacando aquellas relacionadas con la competencia evaluadora. El problema con se encuentra el estudiante de cualquier disciplina está relacionado con varios factores concurrentes que dificultan alcanzar no sólo estos objetivos básicos, sino igualmente los más sofisticados relacionados con el pensamiento crítico y evaluativo en el uso de la información (Leu y Kinzer, 2000). Estos factores son el crecimiento exponencial de toda la información disponible, la dispersión de la misma en múltiples soportes y formatos, sobre todo con el desarrollo de internet (Alvermann, Swafford y Montero, 2004), y su infrautilización debido a carencias formativas o a las dificultades de acceso inherente a algunos tipos documentales (Baildon y Baildonm, 2008).

Así pues, como paso ineludible en cualquier proceso de formación y aprendizaje, está el conocimiento y dominio de las habilidades y destrezas relacionadas con el uso y aprovechamiento de la información y de las tecnologías, englobado bajo el paradigma de competencia información o alfabetización informacional. En el aprendizaje por competencias, el estudiante se convierte en protagonista activo de un aprendizaje virtual, interactivo, compartido y distribuido. La adquisición de destrezas en habilidades relacionadas con el uso y evaluación de los recursos de información le permitirá afrontar las competencias específicas de su área de conocimiento. Este cambio del proceso educativo, que supone el paso de una educación centrada en la enseñanza a una educación centrada en el aprendizaje, implica un nuevo enfoque en el papel de los educadores y de las actividades educativas, y da un mayor énfasis a los resultados de aprendizaje. El aprendizaje basado en competencias refleja la capacidad del estudiante para poner en práctica de manera integrada habilidades, conocimientos y actitudes para enfrentarse y poder resolver problemas y situaciones. Además, permiten evaluar su grado de preparación, suficiencia y responsabilidad.

Este aprendizaje basado en competencias cuenta con interesantes antecedentes como los desarrollados en los proyectos DESECO (2002), 
TUNING (2003), Alfin-EEES (Pinto y García, 2007) e INFOLITRANS (Pinto y Sales, 2008)

Nuestro enfoque del aprendizaje parte de estas premisas básicas (Comunidad Europea, 2003):

- Aprender es un proceso significativo que ocurre a lo largo de toda la vida, y que se extiende en múltiples espacios, tiempos y formas. Está vinculado a las experiencias vitales y a las necesidades de los individuos, así como a su contexto sociocultural.

- Aprender supone el tránsito de la dependencia del individuo a la independencia, de la regulación externa a la autorregulación. El proceso de aprendizaje posee tanto componentes intelectuales como afectivos: se construyen conocimientos, se adquieren destrezas, capacidades y habilidades, pero también se adquieren y modelan valores, convicciones...

- Siendo el centro del aprendizaje el individuo que aprende, aprender es un proceso de participación y de interacción, que fomenta el espíritu reflexivo y la evaluación crítica para solucionar problemas y tomar decisiones.

- El aprendizaje conlleva la interacción del estudiante con los contenidos. En la actual sociedad de la información, con un claro protagonismo de las TICs, va incrementándose la modalidad de enseñanza online, con un claro protagonismo de la virtualidad, la hipertextualidad, la interacción y el uso de recursos educativos electrónicos disponibles principalmente en la web. Estos últimos caracterizados por la inmaterialidad, interactividad, instantaneidad e interconexión, han abierto nuevas posibilidades y ventajas como el incremento considerable de la cantidad de información disponible, la posibilidad de intercomunicación, el intercambio de opiniones, la crítica y el debate en tiempo real. Favorecen enormemente el autoaprendizaje y aportan al estudiante una gran diversidad de funciones. No sólo transmiten información, sino también evaluación, modelización, simulación, etc.

Para que el aprendizaje en entornos virtuales sea realmente significativo, debe de cumplirse estas premisas: de una parte el tutor deberá disponer de habilidades cognitivas que potencien el pensamiento crítico y la capacidad de aprender a aprender; y, de otra, los recursos educativos ofertados habrán de cumplir un estándar de calidad adecuado para que favorezca el aprendizaje.

Con la utilización creciente de las TIC en la enseñanza, la demanda de contenidos educativos para el aprendizaje ha ido creciendo de forma paralela y progresiva, de manera que la producción de los recursos educativos electrónicos es actualmente muy abundante. La variedad y tipología de los mismos es, como podemos imaginar, inmensa, en función de los contextos educativos, de los tipos de actividad, de las aplicaciones y los formatos utilizados o de las plataformas o entornos virtuales de aprendizaje en donde se desarrollen este tipo de materiales. Tal variedad constituye una dificultad a la hora de diseñar estándares que permitan la compartición y la interoperabilidad de recursos educativos, que es la tendencia actual en este ámbito y sobre la que se están proporcionando diferentes soluciones, especialmente a nivel de instituciones y organismos educativos. Pero es que la heterogeneidad se manifiesta desde los inicios de modo que se observa en la literatura cuando se trata de definir qué se entiende por recurso educativo electrónico, o incluso a la luz de las diversas y variadas formas de denominar a estos productos. Parece existir una falta de consenso sobre la definición concreta y la descripción de los recursos educativos electrónicos, aunque se opta por utilizar de forma más generalizada el concepto de "objeto de aprendizaje", objeto educativo u objeto digital educativo (Fernández Manjón et al., 2007), traducción del inglés Learning Object (LO), como unidad básica a partir de la cual pueden construirse estructuras y elementos de contenido más complejos. Esta concepción de Objeto de Aprendizaje (OA) está basada en el paradigma de la Programación Orientada por Objetos, y se plantea como un modelo normalizado para la producción de contenidos y recursos educativos modulares, que puedan ser reutilizados en diferentes contextos y en distintas plataformas tecnológicas (Correa, 2006). Las definiciones sobre OA son amplias y variadas. Apuntamos algunas de ellas:

El Institute for Electrical and Electronic Engineers (IEEE, 2001) define el objeto de aprendizaje como "cualquier entidad, digital o no digital, que puede ser usada, reutilizada o referenciada durante un proceso de aprendizaje apoyado por la tecnología". Wiley (2002) intenta refinar esta definición que considera un tanto extensa y apunta que un $O A$ es "cualquier recurso digital que pueda ser reutilizado como soporte para el aprendizaje", resaltando las características de reusabilidad, adaptabilidad y escalabilidad de los OA (2002). Polsani (2003) redunda en la característica de reusabilidad: "una unidad independiente y auto contenida de contenido de aprendizaje que esta predispuesta a ser reutilizada en múltiples contextos de aprendizaje". Y para López Guzmán (2005) un OA es "cualquier recurso con una intención formativa, compuesto 
de uno o varios elementos digitales, descrito con metadatos, que pueda ser utilizado y reutilizado dentro de un entorno e-learning".

Estas y otras definiciones son muestra de las condiciones que caracterizan básicamente a los objetos de aprendizaje: 1) son reutilizables porque pueden ser modificados y reutilizados en diferentes contextos, 2) son interoperables, porque pueden operar en diferentes plataformas tecnológicas; 3) son durables, pues son flexibles y fáciles de actualizar; y 4) son accesibles, pues son fáciles de localizar y recuperar en una base de datos o en cualquier sistema de almacenamiento de contenidos educativos, gracias a estar normalizados por los metadatos.

Resulta evidente la preocupación por establecer criterios normalizados para la producción de contenidos educativos electrónicos que posibiliten la interoperabilidad y la reutilización de los mismos, con vistas no sólo a un ahorro de tipo económico, sino sobre todo de esfuerzos y de dispersión. Por este motivo, muchas instituciones y organismos se han ocupado de definir estándares para normalizar los OA y establecer un lenguaje común para permitir la comunicación y la interoperabilidad entre las distintas plataformas técnicas que los gestionan. Ello se consigue gracias a la estandarización de metadatos que identifican, describen y clasifican los objetos de aprendizaje. Pero también en este caso, encontramos una gran variedad de estándares. Por ejemplo, Learning Object Metadata (LOM) es el modelo de Metadatos para la descripción de los recursos de aprendizaje propuesto por el IEEE. ARIADNE (Alliance of Remote Instructional Authoring and Distribution Networks for Europe) trabaja en la creación de metodologías para el desarrollo, gestión y reutilización de contenidos educativos, la definición de curricula y metadata educativos. Advanced Distributed Learning (ADL), una iniciativa del Departamento de Defensa de los Estados Unidos que trabaja en colaboración con otras muchas organizaciones, es la impulsora del estándar de e-learning internacional SCORM (Shareable Content Object Reference Model), un modelo de agregación de contenidos que define la manera de especificar la organización interna de los Objetos (estructura), el etiquetado de los Objetos (metadatos), y el modo de empaquetarlos con el fin de permitir su interoperabilidad en diferentes sistemas y plataformas tecnológicas (Correa, 2006). O la iniciativa de Dublin Core Metadata (DCMI) que trabaja también en el desarrollo de estándares de metadatos que faciliten la interoperabilidad. En definitiva, los Objetos de Aprendizaje y su descripción por medio de metadatos estandarizados suponen un gran avance hacia la sistematización en el desarrollo y producción de contenidos educativos, aunque no exista unanimidad en los modelos de metadatos utilizados en los repositorios de contenidos educativos de las instituciones que los mantienen.

En cualquier caso, la tendencia a compartir y a crear recursos educativos abiertos y accesibles a todos aumenta de manera progresiva, y se conforma un nuevo término, el Recurso Educativo Abierto (Open Educational Resource, OER) entendido como aquellos "materiales en formato digital que se ofrecen de manera gratuita y abierta para educadores, estudiantes y autodidactas para su uso y re-uso en la enseñanza, el aprendizaje y la investigación" (UNESCO, 2002). Los nuevos escenarios educativos propician una demanda creciente de este tipo de recursos abiertos, modulares, flexibles, adaptables y de calidad en todos los niveles y áreas de la enseñanza, incluida la universitaria, que puedan ser utilizados y adaptados a la diversidad de circunstancias y contextos didácticos. Por esta razón, se están llevando a cabo por parte de diferentes instancias, tanto públicas como privadas, iniciativas y actuaciones para ofrecer recursos educativos de forma abierta a través de Internet. Es el movimiento conocido como "open courseware" (OCW) o repositorios de OER, cuya filosofía de compartir el conocimiento gracias a las posibilidades proporcionadas por la evolución de la web en los últimos años, está teniendo una gran repercusión en todos los ámbitos. La institución más representativa en este caso es el Massachussets Institute of Technology (MIT) que en 2001 pone en marcha su proyecto de almacenar y difundir de manera pública y abiertos sus contenidos docentes universitarios, con el propósito más ambicioso de crear un movimiento flexible basado en un modelo eficiente que otras universidades puedan emular a la hora de publicar sus propios materiales pedagógicos generando sinergias y espacios de colaboración. $Y$ efectivamente, el OCW del MIT se ha convertido en un ejemplo seguido por muchas otras instituciones universitarias, y se ha conformado un consorcio denominado OCW Consortium (http://www. ocwconsortium.org/) que engloba a más de cien instituciones universitarias de América, Europa, África y Asia, interesadas en la producción y compartición de recursos educativos abiertos de forma normalizada. En España, Universia ha decidido participar en esta iniciativa y crear un OCW entre universidades españolas e iberoamericanas (http://ocw.universia.net/es/).

En el caso de los OCW, los recursos u objetos de aprendizaje son todos los materiales docentes que los profesores universitarios utilizan en 
sus prácticas educativas, y que pueden ser programas docentes, ejercicios y actividades propuestas en clase, vídeos, y demás documentos de cualquier formato utilizados en clase. La definición de OCW propuesta por el MIT es la de un espacio web que contiene materiales docentes creados por profesores para la formación superior, que representan un conjunto de recursos (documentos, programa, calendario,...) utilizados en el proceso de enseñanzaaprendizaje de las asignaturas que imparten los profesores, que se ofrecen libremente y que son accesibles universalmente en la red. Su difusión no se encuentra limitada por cuestiones relativas a la propiedad intelectual, permitiéndose el uso, la reutilización, la adaptación y la distribución por otros, con ciertas restricciones (Creative Commons). Así pues, aunque el MIT presenta una serie de categorías de elementos definidos para describir las asignaturas, éstos no son obligatorios, de forma que los docentes pueden elegir los elementos descriptivos que consideren adecuados para sus cursos (Pernías y Marco, 2007). En cualquier caso y a pesar de estas diferencias entre asignaturas, los OCW constituyen un conjunto homogéneo válido para poder evaluar su calidad.

El papel que deben de desempeñar los recursos educativos electrónicos en el proceso de enseñanza-aprendizaje puede quedar resumido en las siguientes funciones (Pinto, 2009):

- Motivadora: posibilitan un mayor acercamiento a la realidad y ofrecen un contenido más real, que ayude a salir a la enseñanza del puro verbalismo, generando un efecto positivo y motivador que favorecerá un ambiente de aprendizaje más favorable.

- De apoyo para la presentación del contenido: Es una de las funciones fundamentales de los medios y recursos digitales al convertirse en un pilar de apoyo favorecedor de los procesos de aprendizaje que permiten alcanzar los objetivos propuestos.

- Estructuradora: Es la capacidad metodológica que tienen los medios electrónicos para guiar las actividades del estudiante y facilitar determinadas experiencias de aprendizaje. En este sentido, la enseñanza en línea, y los recursos electrónicos a ella asociados puede ponerse como ejemplo de un medio de máximo poder estructurador en lo metodológico.

\section{La necesidad de instrumentos evaluadores}

Se sabe que un adecuado control de la calidad en la producción, presentación y acceso de los entornos y recursos digitales favorece los procesos de enseñanza-aprendizaje permitiendo una mayor implicación de la comunidad educativa universitaria en el conjunto del proceso educativo. Cuanto mayor es la calidad de los recursos que las universidades ponen a disposición de los estudiantes mayor es también su visibilidad y presencia en el contexto científico nacional e internacional.

La evaluación de la calidad de los recursos de aprendizaje virtual es una necesidad ineludible que choca con la dificultad que plantea su crecimiento exponencial. A las características que debe tener cualquier recurso digital se suman las específicas de los recursos educativos, más centradas en el aprendizaje y en la adquisición de competencias.

Los criterios básicos de calidad de los recursos de información digital en general y de los recursos educativos digitales en particular ha sido ampliamente abordada en la literatura, aunque se aprecia una gran dispersión en su valoración y uso. (Shneiderman, 1998; Nielsen, 1993; Marzal, 2008).

El término calidad, aplicado a la información digital, es una meta que implica un proceso continuo de planificación, análisis, diseño, implementación, promoción e innovación, para asegurar que la información cubra las necesidades de formación y aprendizaje de los usuarios en cuanto a contenido, presentación y usabilidad. Sin embargo, no todos los productores de información apuestan por la calidad debido al esfuerzo que ello requiere y por eso encontramos en internet recursos de todo tipo. Si a esto le sumamos la naturaleza caótica de la red, que dificulta la búsqueda, la identificación y la localización de la información deseada veremos que se hace necesario conocer las características que definan la calidad de los recursos educativos electrónicos, para poder valorarlos y filtrarlos. De ahí que la valoración y determinación de su calidad sea un elemento importante para profesores, educadores, estudiantes e instituciones educativas (MEC, 1995).

Por tanto, aplicadas las definiciones de calidad al ámbito de la información podríamos decir que la calidad de la información de un recurso educativo electrónico vendrá determinada por su capacidad para satisfacer las necesidades de información de los estudiantes y profesores que lo utilicen o consulten. Si bien, esto puede ser 
muy relativo, ya que la apreciación de la calidad es subjetiva y lo que para una persona puede ser válido puede no serlo para otra, lo cierto es que hay una serie de convenciones universalmente aceptadas de las dimensiones deseables de la información de calidad que podemos agrupar en estas cuatro categorías: intrínseca, contextual, representacional y accesibilidad.

La calidad intrinseca hace referencia a la calidad de la información per se, a su valor objetivo independientemente de su forma de difusión, diseño o al público al que va dirigido. Se engloban aquí aspectos como:

- Rigor científico: Es importante que la información que aporte un documento esté basada en la evidencia científica, en la reflexión y en los métodos científicos propios de cada disciplina.

- Integridad: La información no debe ser parcial ni sesgada, sino que debe presentarse en su totalidad a no ser que la finalidad del documento sea resumir o abreviar otra información.

- Objetividad: Depende del autor de la misma y no de la percepción del estudiante. La credibilidad de la información se asocia a la confianza que nos merezca el responsable de su contenido en función de su autoridad y su afiliación académica.

- Precisión: Tiene que ver con la exactitud de la información y con el nivel de profundidad con que se aborda un tema. No obstante, esta dimensión depende de la intención y las pretensiones del recurso y del tipo de estudiante al que va dirigida.

La calidad contextual está relacionada con el contexto en el que se accede a la información y con la adecuación a las necesidades del sistema y del estudiante.

- Relevancia: Sería la adecuación de la información a las necesidades de los estudiantes. Es por tanto una dimensión de valoración subjetiva condicionada al tipo de estudiante que la usa.

- Valor añadido. En muchas ocasiones los elementos de valor añadido facilitan el uso de la información y permiten una mejor asimilación de la misma, aumentando su utilidad y calidad.

- Actualidad de la información: Determina su utilidad.

- Utilidad: Responde a la pregunta ¿para que sirve esta información? Aunque depende de para qué quiere ese estudiante esa informa- ción, es evidente que también hay un componente objetivo relacionado con la finalidad de la información y el perfil de estudiante al que se dirige.

- Adecuación: es importante saber a qué estudiantes va dirigida la información a la hora de divulgarla y adecuarla a ese perfil.

La calidad representacional trata de la forma en que se representa la información, así como de todos los aspectos técnicos referidos a su estructura. Se deben analizar estos aspectos: tipo de formato, claridad, concisión, compatibilidad, diseño, homogeneidad de los datos...

La calidad del acceso engloba los aspectos relativos al cómo se accede a la información, tales como tiempo de espera, navegación y seguridad.

Con el incremento del uso de las TIC en la educación y en programas e-learning, la evaluación y la mejora por la calidad de los recursos digitales educativos disponibles en Internet se ha convertido en un frente de investigación importante y a la vez un reto que tienen que afrontar los organismos públicos competentes, como sucede tanto en países de la Unión Europea: Portugal, Francia, Gran Bretaña o Italia, como de Norteamérica: Canadá y Estados Unidos. Cualquiera de ellos ha desarrollado marcas de reconocimiento que avalan la calidad de los recursos educativos electrónicos con fines de aprendizaje a lo largo de la vida, tanto a nivel de sus contenidos, del acceso y navegabilidad como de la ergonomía.

La evaluación de los recursos educativos electrónicos ha sido objeto de estudio a nivel internacional, se han propuesto pautas, se han establecido diferentes metodologías y el elenco de criterios e indicadores de evaluación es bastante notable. De hecho en la literatura podemos encontrar tanto trabajos de carácter teórico (British Columbia, 2002; Branch, Kim, Koencke, 2000; Reinders, Lewis, 2006; Hosie, Schibeli, 2005), como análisis concretos de casos (Markland y Kemp, 2004; Lind, 2005; Li, Nesbit, Richards, 2006). En España no son muchos los estudios y generalmente abordan este tema desde un enfoque parcial, centrado en algunos tipos de recursos o en algunas disciplinas (Area, 2002; de Pablos, 2002; Marquès Graells, 2005; Marzal et al., 2006, Cuevas Cervero, 2003; Díaz et al. 2002; Díaz, 2003; Padrón, 2009).

La usabilidad como disciplina que estudia la forma de facilitar la interacción entre las personas y el ordenador, de manera que los usuarios puedan usar los sitios web de manera fácil, cómoda e intuitiva, es el enfoque más generali- 
zado en la literatura científica para evaluar la calidad de los recursos educativos electrónicos. La usabilidad, o más bien, la evaluación de la usabilidad, ha ido incorporando matices relativos a diversos campos de estudio afines como el diseño de la interfaz centrada en el usuario, las ciencias de la computación, las ciencias de la conducta, la psicología cognitiva, las comunidades virtuales, entre otras, de manera que lo que tradicionalmente había sido su esfera de acción en el ámbito Interacción PersonaOrdenador $(\mathrm{HCl})$ se ha ido ampliando a medida de los años, incorporando elementos de reflexión acerca de la experiencia de los usuarios, sus necesidades y expectativas, el contexto, etc. (Preece et al., 2002). Cuando la usabilidad es aplicada en el ámbito del e-learning, los requerimientos de calidad engloban elementos específicos derivados de los procesos de enseñanza-aprendizaje. Es decir, no sólo se valoran aspectos relacionados con los elementos técnicos y el diseño de la interfaz web, sino también los elementos pedagógicos que aseguren la eficacia y el logro de los objetivos docentes propuestos. La necesidad de integrar las nociones clásicas de usabilidad y aprendizaje es puesta de manifiesto por Squires (1999), al condicionar la calidad de un recurso de e-learning no sólo al carácter "usable" de su diseño sino también a su valor pedagógico. Algunos otros autores señalan que un recurso de aprendizaje electrónico puede ser muy válido desde la perspectiva de la usabilidad técnica, pero puede no tener ningún valor pedagógico, y a la inversa (Quinn, 1996; Albion, 1999; Squires y Preece, 1996). Esta dimensión de la usabilidad, denominada usabilidad pedagógica o instruccional, se incorpora en el desarrollo y diseño de métodos de evaluación y valoración de la calidad de los recursos educativos electrónicos, ampliando los criterios clásicos de la usabilidad (Reeves, 1997; Laurillard, 2002; Nokelainen, 2006).

Las últimas tendencias incorporan las dimensiones afectivas del aprendizaje a la evaluación de las aplicaciones de e-learning. En el ámbito del e-learning y de la interacción personacomputador, el análisis de los estados afectivos se convierte en una disciplina emergente (Picard et al., 2004), que tiene como objetivo hacer participar a los estudiantes en una experiencia de aprendizaje exitosa promoviendo estados afectivos positivos. Zaharias (2009) argumenta que la "motivación para aprender" es el factor de aprendizaje afectivo que más puede influenciar la interacción de los usuarios con la aplicación e-learning, y propone esta motivación como una nueva medida de usabilidad para el diseño de elearning.

\section{El proyecto EVALUAREED}

Para profundizar en este tema hemos desarrollado el proyecto I+D EVALUAREED (SEJ2007.62244) con un enfoque novedoso en la medida en que pretende, en primer lugar, hacer un análisis cuantitativo y cualitativo de los recursos educativos electrónicos en las universidades españolas, describiendo las distintas tipologías y sus características; en segundo lugar, basándonos en un exhaustivo estudio de la literatura científica ofrecer pautas concretas para evaluar la calidad de los distintos tipo de recursos digitales, con especial atención a una de esas tipologías, los OCW; y, en último lugar, elaborar un software que ayude a la identificación de su calidad y oriente sobre cómo mejorar el recurso.

El resultado final se concretará en el desarrollo de un portal sobre evaluación de recursos electrónicos educativos que contará con una herramienta automática basada en un checklist, que ayude a la identificación de la calidad de los recursos educativos electrónicos de dominio público presentes en las universidades españolas, comprobando su nivel de calidad, destacando las fortalezas y los puntos de mejora.

\subsection{Metodología}

Se parte de un enfoque cualitativo-cuantitativo para llegar al establecimiento de una matriz de análisis formada por criterios e indicadores ponderados, que se aplican a una muestra representativa de recursos con el objeto de determinar su eficacia y eficiencia y sus diferentes contextos y condiciones de uso. Los indicadores son de dos tipos: estimados por expertos humanos y programados en una sonda automática. Se presta especial atención a la relación entre ambos y a su complementariedad de cara a permitir una evaluación asistida por ordenador lo más objetiva posible aunque sin prescindir del juicio humano en aquellos aspectos en que éste sea necesario. La creciente incorporación de factores relacionados con el prestigio del responsable, la actualización, la estructuración del contenido, la correlación entre contenidos de la página y del título, etc. y, por supuesto, la cita electrónica, en los motores de búsqueda, muestra cómo la incorporación de indicadores cuantitativos de la calidad del recurso empieza a ser un criterio de uso creciente en la recuperación de recursos digitales, y apoya la aproximación propuesta en este estudio a la evaluación de los recursos y plataformas de aprendizaje. 


\subsection{Fases}

La metodología utilizada aborda las siguientes fases: la identificación y selección de los recursos a evaluar, el establecimiento de los criterios de evaluación con la creación del checklist y la creación del portal EVALUA-REED.

\subsubsection{Identificación y selección de recursos a evaluar.}

En bastantes ocasiones se solventa esta cuestión mediante la selección de una muestra aleatoria lo suficientemente amplia. Esto viene dado por dos factores: el desconocimiento de la totalidad de los recursos existentes, y la imposibilidad de tratar de forma manual cantidades grandes de recursos. Sin embargo, la disponibilidad (y la experiencia en este campo) de herramientas y técnicas automáticas de localización y extracción de datos, permite abordar este tema desde la perspectiva de una selección lo más exhaustiva posible de recursos de aprendizaje en el dominio universitario español.

De otro lado el carácter hipermedia de este tipo de recursos implica la interconexión de muchos de tales recursos entre sí, y con recursos de otro tipo también. El estudio de este tipo de interconexiones podría aportar informaciones de interés en este campo. Pero no es posible abordar este tipo de estudio limitándose a una simple muestra, $y$, si bien no es realista pensar que se van a localizar todos los recursos de este tipo existentes, si creemos estar en condiciones de localizar mediante herramientas automáticas una cantidad suficiente de recursos en función de criterios de cita y otros elementos objetivos.

\subsubsection{Identificación de criterios y de la forma de la toma de datos}

Partiendo de los criterios y metodologías propuestos en la literatura para evaluar recursos digitales, se haría una selección de aquellos que sean más adecuados para la evaluación de cada tipo de recurso. Esta etapa es especialmente importante puesto que en la literatura se han propuesto muchos de carácter general (por ejemplo, Merlo Vega, 2003; Saracevic, 2002, Bargheer, 2003; Place et al., 2006; Lee, et al., 2002) y no todos son aplicables a todos los tipos de recursos educativos. Se diseña una plantilla para la recogida de información. Dependiendo del aspecto a evaluar en algunos casos será posible utilizar herramientas automáticas para la extracción de información, mientras que en otros será imprescindible la recolección manual de información por parte del equipo investigador, puesto que se requerirá una valoración de experto. Para determinados indicadores se utili- zará el robot Sacarino para tomar datos del tipo volumen, tamaño, formatos, número de enlaces activos, número de enlaces erróneos, número y tipo de metadatos, visibilidad o impacto; mientras que para otros tendremos que acceder al recurso y observar y valorar otra serie de aspectos, como por ejemplo presentación, accesibilidad, organización de la información, actualidad de la información, autoría o navegabilidad.

\subsubsection{Análisis de los datos.}

Se procede al análisis cualitativo-cuantitativo de los datos con el fin de obtener un diagnóstico de la situación general de los recursos educativos electrónicos, modalidad OCW, en el conjunto de las universidades españolas, teniendo en cuenta las especificidades de las universidades y de los ámbitos temáticos estudiados, con el fin de establecer las mejores prácticas en cada ámbito y las principales carencias, así como sus similitudes y diferencias a través de técnicas de escalamiento multidimensional y análisis de clusters. Para tener un conocimiento mayor de la situación se observará qué factores son los que más influyen y marcan la diferencia entre la calidad de unos recursos y otros a través del Análisis de Componentes Principales y Análisis Factorial.

\subsubsection{Creación del portal EVALUA-REED.}

Para poder dar a conocer los resultados obtenidos y filtrados, se creará un portal en el que se recojan:

- Enlaces y documentos referentes a la creación y evaluación de recursos educativos en formato digital adaptados a la enseñanza universitaria.

- Tipología de recursos educativos digitales.

- El checklist EVALUAREED, pilotado y validado.

- Los resultados detallados de la investigación.

- Las mejores prácticas y los defectos más comunes en este tipo de recursos, así como un buen número de ejemplos para ilustrarlo.

- Tutoriales que ayuden al usuario a detectar la calidad de los recursos y plataformas y que sirvan de guía a las personas interesadas en su diseño o creación.

- Diseño del checklist EVALUAREED (versión piloto), que comprende un conjunto de criterios y subcriterios para evaluar la calidad de los recursos educativos. Esta herramienta automática permitirá evaluar la calidad de un recurso a partir de los datos introducidos y 
ge nerará un informe sobre su calidad, los puntos débiles y la manera de mejorarlos

A continuación, se detalla la propuesta del checklist EVALUAREED. Para cada ítem, se responde sí o no y, si lo hay, se pueden añadir comentarios.

1. Calidad del contenido: ¿Es el contenido apropiado y con nivel de detalles?

- Contenido libre de errores.

- Contenido apropiado y actualizado.

- Contenido suficientemente detallado para alcanzar los objetivos de aprendizaje.

- Materiales bien organizados y estructurados.

- Contenido secuenciado de manera lógica y efectiva.

- Actividades suficientes en número y en posibilidades para conseguir los objetivos de aprendizaje.

- Existencia de recursos multimedia (apropiados).

- Existencia de enlaces externos a información relevante, apropiada y actualizada.

- Existencia de sección de FAQs.

- El vocabulario y la terminología usados son apropiados para los alumnos.

- Relevancia de los materiales de referencia.

2. Objetivos de aprendizaje: ¿Están clara y sistemáticamente indicados el contenido y las actividades.

- Los objetivos, aprendizaje, contenido y actividades de evaluación son sistemáticos.

- Los objetivos de aprendizaje son claros y explícitamente indicados.

3. Feedback o motivación: ¿Existen posibilidades de feedback para el alumno?.

- El feedback apoya resultados de aprendizaje positivo.

- Se proporciona al alumno respuestas correctivas en tiempo, respuestas a preguntas e información.

- El feedback usa un lenguaje que motiva al alumno al aprendizaje y al esfuerzo.

- Si el objetivo es interactivo, se proporciona feedback a las acciones de los usuarios.

4. Efectividad: ¿Mejora el recurso el aprendizaje y la docencia?.
- El contenido es relevante para conseguir los objetivos de aprendizaje.

- El recurso proporciona oportunidades atractivas de aprendizaje.

- El recurso posee múltiples estilos de aprendizaje: audio, imágenes, video y texto.

- El contenido trata importantes, valiosos y significativos conceptos, modelos o habilidades educativas.

- Estimula habilidades de comunicación.

- Anima a la interacción del grupo.

- Estimula la creatividad del estudiante.

- Permite/estimula al estudiante a trabajar de forma independiente.

- Se proporcionan herramientas apropiadas de evaluación.

- Se incluyen actividades individuales y actividades basadas en grupo.

- Se ofrecen instrumentos (libreta de notas, ayudas al trabajo, recursos, glosario, etc.) que apoyan el aprendizaje.

- Los documentos que se descargan se ven correctamente.

- Los documentos y la información del curso se pueden encontrar y localizar fácilmente.

5. Usabilidad: ¿Es el diseño y la funcionalidad de la interfaz del usuario sistemática y predecible?.

- Los elementos comunes, los botones de navegación y el texto están sistemáticamente situados.

- El diseño es claro, sistemático y proporciona instrucciones para la navegación.

- Los elementos de navegación proporcionan la posibilidad de play, replay, stop y control del volumen.

- Los hipervínculos, los botones y demás herramientas de navegación funcionan correctamente.

- Los elementos gráficos tienen propósitos educativos claros.

- El contenido puede ser visto con un scrolling mínimo.

- Existencia de una página modelo normalizada.

6. Reusabilidad: ¿Se puede utilizar el contenido de aprendizaje en otros contextos de aprendizaje?. 
- Los requisitos del software están identificados y no tienen ningún coste.

- El recurso contiene todos los recursos necesarios para completar la actividad y evita dependencia externa.

7. Accesibilidad: ¿Es el diseño accesible a todos los alumnos?

- El recurso cumple los requisitos de accesibilidad de la WAI.

- El recurso está libre de problemas técnicos (enlaces erróneos, errores de programación).

8. Propiedad intelectual y copyright: ¿Están los derechos de compartir el recurso y de autoría indicados explícitamente en el recurso o en los metadatos?.

- El permiso para utilizar materiales con copyright ha sido obtenido.

- El copyright está claramente indicado e incluye información de contacto con los titulares del mismo.

- Si el contenido ha sido desarrollado y pertenece a la persona que presenta el recurso, se adjunta una licencia Creative Commons o similar.

\section{Conclusiones}

La creación y diseño de recursos educativos electrónicos, su almacenamiento en repositorios abiertos y la compartición y el intercambio de los mismos es una tendencia que se incrementa progresivamente en los momentos actuales en todos los niveles de la educación. La utilización de los mismos en las prácticas docentes es ya una realidad que cada vez va adquiriendo más peso, especialmente en la educación universitaria, que además está sufriendo una transformación importante en sus ideas respecto a la disponibilidad y acceso a los materiales docentes. Ello es evidente a la luz de las iniciativas OCW de creación de repositorios de materiales de asignaturas universitarias, de acceso abierto y público. Por lo tanto, resulta normal que el estudio y la revisión de la literatura científica nos ofrezca un interés constante por indagar estos aspectos con el objetivo de encontrar criterios de calidad en la producción y diseño de los materiales docentes, así como por establecer modelos de evaluación que permitan unos recursos educativos útiles, válidos, fiables, reutilizables e intercambiables. La heterogeneidad en la definición de estos criterios de calidad es paralela a la existente a la hora de delimitar el concepto de objeto o recurso de aprendizaje, dada la amplísima variedad de objetos digitales que pueden ser considerados recursos educativos, y la evolución constante de la tecnología, que nos hace movernos en un escenario móvil en el que los límites son difíciles de fijar.

Se pretende que el portal y el checklist EVALUAREED sean de gran utilidad a la comunidad científica universitaria en unos momentos de significativo cambio, ofreciendo una metodología contrastada para evaluar y determinar la calidad de los recursos y plataformas educativas electrónicas. La mejora de los recursos y entornos de aprendizaje permite la creación de redes virtuales de conocimiento compartido $\mathrm{y}$, por tanto, la mejora del conocimiento.

\section{Notas}

(1) Este trabajo es resultado del proyecto de I+D financiado por el Ministerio de Ciencia e Innovación (SEJ 2007.62244).

\section{Referencias}

Albion, P. R. (1999). Heuristic evaluation of educational multimedia: from theory to practice. // Proceedings of 16th Annual conference of the Australasian Society for Computers in Learning in Tertiary Education, ASCILITE, 1999.

Alvermann, D. E.; Swafford, J.; Montero, M. K. (2004). Content area literacy instruction for the elementary grades. Upper Saddle River, NJ: Pearson Education, 2004.

Area, Manuel; Castro, Fátima; De La Cruz, Alicia; Sanabria, Ana; Estévez, Román (2002). Análisis De webs docentes de tecnología Educativa y Nuevas Tecnologías aplicadas a la educación de las universidades españolas. // $X$ Jornada del profesorado de Tecnología Educativa. Barcelona, 2002. Presentación

Baildon, R.; Baildon, M. (2008). Guiding Independence: Developing a Research Tool to Support Student Decision Making in Selecting Online Information Sources. // The Reading Teacher. 61:8 (2008) 636-647.

Bargheer, M. (2003). Quality Control and Evaluation of Scientific Web Resources. // Bibliothek. 27:3 (2003) 153169

Branch, R.; Kim, D.; Koenecke, L. (2000) Online educational materials for use in instruction. // Teacher Librarian. 28:1 (2000) 21-23.

British Columbia, Ministry of Education. (2002). Evaluating, selecting and managing learning resources. http://www.bced.gov.bc.ca/irp/resdocs/esm guide.pdf (2010-03).

Comunidad Europea (2003). El papel de las universidades en la Europa del Conocimiento. Bruselas, 2003. http://www.crue.org/export/sites/Crue/procbolonia/docum entos/antecedentes/7._El_papel_de_las_universidades. pdf (2010-04)

Correa, L. F. (2006). El confuso universo de los Objetos de Aprendizaje. http://www.universia.net.co/index2.php? option $=$ com_content\&do_pdf=1\&id=5082 (2010-03).

Cuevas Cerveró, A.; Calzada-Prado, J.; Colmenero Ruiz, M.J. (2003). Recursos educativos en Internet: portales educativos. // Congreso Internacional Virtual de Educación, 1-11 de abril 2003.

De Pablos; J.; García, R.; Barragán, R.; Buzón, O. (2002). Análisis de páginas web elaboradas por docentes de 
Tecnología educativa: una aproximación descriptiva desde conceptos socioculturales. Grupo de investigación, evaluación y tecnología Educativa, Facultad de Ciencias de la educación, Universidad de Sevilla, 2002.

Díaz, P.; Sicilia, M. A.; Aedo, I. (2002). Evaluation of hypermedia educational systems: Criteria and imperfect measures. // International Conference on Computers in Education (ICCE 2002). Auckland (Nueva Zelanda). IEEE Press. 621-626.

Díaz, P. (2003). Usability of hypermedia educational ebooks. I/ D-Lib Magazine. 9:3 (2003). http:// www.dlib.org/dlib/march03/diaz/03diaz.html (2010-03).

Fernández Manjón, B.; Moreno Ger, P.; Sierra Rodríguez, J. L.; Martínez Ortiz, I. (2007). Uso de estándares aplicados a Tic en educación. CNICE. Disponible en http://ares.cnice.mec.es/informes/16/versionpdf.pdf (2010-03).

Hosie, P.; Schibeli, R. (2005). Checklist and Context-Bound evaluations of online learning in higher education. // British Journal of Educational Technology. 36:5 (2005) 881895.

IEEE Learning Technology Standards Committee (LTSC). (2001). Learning Object Metadata Working Group. Disponible en http://www.ieeeltsc.org:8080/Plone (2010-03).

Jonassen, D. (1991). Objetivism versus constructivism: Do we need a new philosophical paradigm? // Journal of Educational Technology Research and Development. 39:3 (1991) 5-14

Laurillard, D. (2002). Rethinking University Teaching: A Conversational Framework for the Effective Use of Learning Technologies. London and New York: Routledge, 2002.

Lee, Yang W.; Strong, Diane M.; Kahn, Beverly K.; Wang, Richard Y. (2002). AIMQ: a methodology for information quality assessment. // Information and Management. 40:2 (2002) 133-146

Leu, D. J., Jr.; Kinzer, C. K. (2000). The convergence of literacy instruction with networked technologies for information and communication. // Reading Research Quarterly. 35:1 (2000) 108-127.

Li, J., Nesbit, J. Richards, G. (2006). Evaluating learning objects across boundaries: the semantics of localization. // International Journal of Distance Education Technologies. 4:1 (2006) 17-30

Lind, L. (2005). Defining the quality of online learning material in Finland. Policy and Innovation Committee Meeting, Birminghan, 2005.

López Guzmán, C. (2005). Los Repositorios de Objetos de Aprendizaje como soporte a un entorno e-learning. Trabajo de Grado. Universidad de Salamanca, 2005. http://www.biblioweb.dgsca.unam.mx/libros/repositorios/ contenido.htm (2010-03).

Markland, M.; Kemp, B. (2004). Integrating digital resources into online learning environments to support the learner. Networking Learning Conference, Lancaster University, England.

http://www.networkedlearningconference.org.uk/past/nlc 2004/proceedings/symposia/symposium9/markland_kem p.htm (2010-03).

Marquès Graells, P. (2005). Las webs docentes. 2005. http://dewey.uab.es/pmarques/webdocen.htm (2010-03)

Marzal, M.A.; Calzada, J.; Viannello, M. (2008). Criterios para la evaluación de la usabilidad de los recursos educativos virtuales: un análisis desde la alfabetización en información. // Information Research, 13:4 (2008) paper 387. http://InformationR.net/ir/13-4/paper387.html (201003).

Marzal, M.A.; García Jiménez, A.; Calzada-Prado, et. al. (2006). Documentación y tecnologías de la información para Educación: herramientas para la alfabetización informacional y la organización de recursos didácticos: Estado del proyecto. $/ / 4^{\circ}$ Simposium Internacional de Bibliotecas Digitales "Información al servicio de la sociedad", Málaga, 21-23 de junio, 2006.

MEC (1995): Calidad de la educación y eficacia de la escuela: estudio sobre la gestión de los recursos educativos. Madrid: Ministerio de Educación y Ciencia, Centro de Publicaciones, 1995.

Merlo, J. A. (2003). La evaluación de la calidad de la información web: aportaciones teóricas y experiencias prácticas. // Recursos informativos: creación, descripción y evaluación. Mérida: Junta de Extremadura, 2003. 101110.

Nielsen, J. (1993). Usability engineering. New York: Academic Press, 1993

Nokelainen, P. (2006). An empirical assessment of pedagogical usability criteria for digital learning material with elementary school students. // Educational Technology \& Society. 9:2 (2006) 178-197.

Organisation de Cooperation et de Developpement Economiques. Définition et sélection des compétences (DESECO) : fondements théoriques et conceptuels. 2002. www.portal-stat.admin.ch/deseco/deseco_doc_strategique.pdf.

Padrón, M. L. (2009). Desarrollo de materiales didácticos desde una perspectiva basada en modelos. Tesis Doctoral dirigida por M. Paloma Díaz Pérez. Escuela Politécnica Superior. Universidad de Carlos III, 2009. http://earchivo.uc3m.es/bitstream/10016/5679/1/Tesis_CL_Padron_Napoles.pdf (2010-04).

Pernías, M.; Marco, M. (2007). Motivación y valor del proyecto OpenCourseWare: la universidad del siglo XXI Contenidos educativos en abierto. // Revista de Universidad y Sociedad del Conocimiento (RUSC). 4:1 (2007). UOC. http://www.uoc.edu/rusc/4/1/dt/esp/pernias marco.pdf (2010-04).

Picard, R. W.; Papert, S; Bender, W; Blumberg, B; Breazeal, C; Cavallo, D; et al. (2004). Affective Learning: a manifesto. // BT Technology Journal. 22:4 (2004).

Pinto, M.; Sales, D.; Bas, N.; Planelles, E. (eds.). Documentación aplicada y Espacio Europeo de Educación Superior. Madrid, Arco Libros, 2009.

Pinto, M.; García Marco, F. J. (2007). La enseñanzaaprendizaje de las competencias genéricas en el Espacio Europeo de Educación Superior: el proyecto ALFINEEES. En: Innovación docente, tecnologías de la información y la comunicación e investigación educativa en la Universidad de Zaragoza: caminando hacia Europa. Zaragoza: Universidad, 2007. p. $62-63+32$ p. en CD. http://www.unizar.es/eees/innovacion06/capitulo2. html (2010-03)

Pinto, M.; Sales, D. (2008). INFOLITRANS: A Model for the Development of Information Competence for Translators. // Journal of Documentation. 64:3 (2008) 413-437.

Place, E.; Kendall, M.; Hiom, D.; Booth, H.; Ayres, P.; Manuel, A.; Smith, P. (2006). Internet Detective: Wise up to the Web, 3rd edition, Intute Virtual Training Suite. http://www.vts.intute.ac.uk/detective/ (2010-03).

Polsani, P. R. (2003). Use and Abuse of Reusable Learning Objects. // Journal of Digital information, 3 (4) http://www.info2.uqam.ca/ nkambou/DIC9340/seances/s eance 10 et $12 /$ Standards $\% 20$ et $\% 20 \mathrm{LO} / \mathrm{http} \_$jodi.ecs. soton.ac.pdf (2010-04).

Preece, J.; Rogers, Y.; Sharp, H. (2002). Interaction Design: Beyond Human-Computer Interaction. New York: John Wiley \& Sons, 2002.

Quinn, C. N. (1996). Pragmatic evaluation: lessons from usability. // Proceedings of 13th Annual Conference of 
the Australasian Society for Computers in Learning in Tertiary Education, (1996). http://www.ascilite.org.au/ conferences/adelaide96/papers/18.html (2010-03).

Reeves, T. C. (1997). Evaluating What Really Matters in Computer-Based Education. http://www.eduworks.com/ Documents/Workshops/EdMedia1998/docs/reeves.html (2010-03).

Reinders, H.; Lewis, M. (2006). An evaluative checklist for self-access materials. // ELT Journal. 60:3 (2006) 272278.

Rumsey, S. (2008). How to find information. MacGraw Hill, 2008. 7-10.

Saracevic, T. (2002). D-Lib Edu: resources for education in digital libraries. http://www.scils.rutgers.edu/ tefko/ D_LibEdu_home.htm (2010-03).

Shneiderman, B. (1998). Designing the user interface: strategies for effective human-computer interaction. Reading: Addison-Wesley, 1998.

Squires, D.; Preece, J. (1996). Usability and learning: evaluating the potential of educational software. // Computers and Education. 27:1 (1996) 15-22.

Squires, D. (1999). Usability and Educational Software Design: Special Issue of Interacting with Computers. Interacting with Computers.11:5 (1999) 463-466.
Tuning, Educational Structures in Europe. Informe Final. Fase Uno. Bilbao: Universidad de Deusto, 2003. http://www.relint.deusto.es/TUNINGProject/spanish/doc2 fase1.asp (2010-03).

UNESCO. (2002). Forum on the impact of open courseware for higher education in developing countries: final report. Paris: UNESCO. http://unesdoc.unesco.org/images/00 12/001285/128515e.pdf (2010-04).

Unión Europea (2004). Elearningeuropa.info. http://www. elearningeuropa.info/ (2010-03)

Wiley, D. A. (2002). Connecting Learning Objects to Instructional Design Theory: A Definition, a Metaphor, and a Taxonomy. D. A. Wiley (ed.), The Instructional Use of Learning Objects. Agency for Instructional Technology and Association for Educational Communications and Technology, 2002. 3-23.

Zaharias, P. (2009). Developing a Usability Evaluation Method for E-learning Applications: From Functional Usability to Motivation to Learn. // International Journal of Human-Computer Interaction. 25:1 (2009) 75-98.

Recibido: 24-05-2010.

Aceptado: 24-05-2010. 